Sir,

\section{Response to Mr Imran Rahman}

Rahman's report of his local, prospective study provides further evidence of the inadequate opportunities to complete full intraocular procedures available to SHOs, especially in these days of extreme pressure to maximise throughput. Once again it demonstrates that this cannot be explained in terms of the pressure of larger list sizes. What is particularly impressive in his report is the way in which this information was used to reverse the situation and substantially increase the opportunities available to SHOs. While we would agree that negative comments on their own do not lead to change, his work shows the importance of listening to the concerns of junior doctors and acting to address them. We hope that the striking success of his department in doing so will encourage other departments to follow their example.

MG Boulton', A Gibson², MP Watson³, MJ Moseley4, PI Murray ${ }^{5}$ and AR Fielder ${ }^{4}$

${ }^{1}$ School of Health and Social Care, Oxford Brookes University, Headington, Oxford, UK

${ }^{2}$ Oxford Eye Hospital, Oxford, UK

${ }^{3}$ Imperial College London, Hammersmith Hospital, London, UK

${ }^{4}$ Department of Ophthalmology, Department of Optometry and Visual Science, City University, London, UK

${ }^{5}$ Academic Unit of Ophthalmology, Division of Immunity and Infection, University of Birmingham, Birmingham, UK

Correspondence: PI Murray,

Tel: + 441215076851 ;

Fax: + 441215076853 .

E-mail: p.i.murray@bham.ac.uk

Eye (2006) 20, 1426. doi:10.1038/sj.eye.6702283; published online 10 February 2006

Sir,

Paradoxical worsening with superior ophthalmic vein thrombosis after gamma knife radiosurgery for dural arteriovenous fistula of cavernous sinus: a case report suggesting the mechanism of the phenomenon

Stereotactic gamma knife radiosurgery is proposed as a safe, less-invasive and effective treatment for low-flow dural arteriovenous fistula of cavernous sinus (DAVFCS). ${ }^{1}$ With the integration of the stereotactic angiographic and magnetic resonance (MR) imaging results during radiation dose planning, gamma knife radiosurgery imposes minimal damage to the adjacent vital structures. ${ }^{2}$ Progressive occlusion of the fistula may impose less haemodynamic impact to the cerebral vascular network compared to endovascular treatment. We report a case of paradoxical worsening of symptoms and signs with superior ophthalmic vein (SOV) thrombosis and central retinal vein occlusion (CRVO) after gamma knife radiosurgery before complete obliteration of the fistula. The MR images depict the mechanism of this phenomenon.

\section{Case report}

A 37-year-old women was diagnosed with DAVFCS and received gamma knife radiosurgery in the other hospital. Angiographic examination showed a left sidetype B DAVFCS, ${ }^{3}$ which was drained into the anterior and inferior compartment of the left cavernous sinus and subsequently into the left SOV (Figure 1). At 1 month after gamma knife radiosurgery, she developed increasing proptosis and vision deterioration in her left eye and was referred to our department.

The best-corrected visual acuity was $6 / 5$ in the right eye and 6/10 in the left eye. Fundus examination and fluorescence angiography disclosed CRVO in the left eye. MR examination revealed a prominent left side cavernous sinus and absence of flow void phenomenon in the left SOV (Figure 2). The abnormal flow void signal at the anterior portion of the left cavernous sinus before treatment was replaced by soft tissue signal, indicating thrombus formation at this portion (Figure $3 a$ and $b$ ). Doppler ultrasonography revealed no flow in the

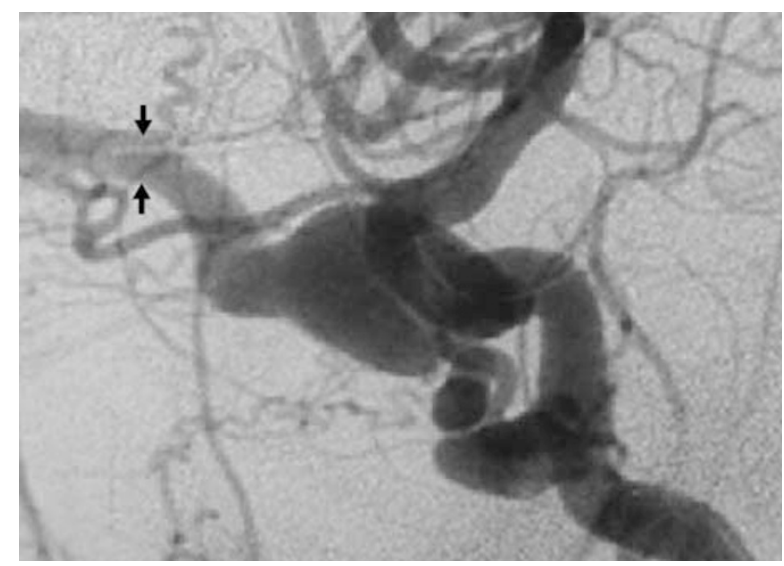

Figure 1 Angiography before gamma knife radiosurgery showed a prominent left superior ophthalmic vein (arrows) draining the anterior compartment of the cavernous sinus. 


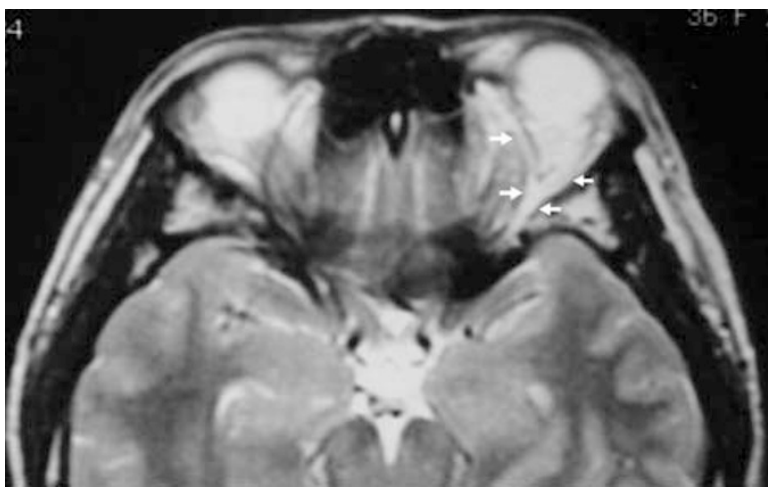

Figure 2 MR T2-weighted image 1 month after gamma knife radiosurgery revealed absence of flow void phenomenon in the engorged left superior ophthalmic vein (arrows). Tissue density of the lumen indicating thrombus formation in the left superior ophthalmic vein is shown.
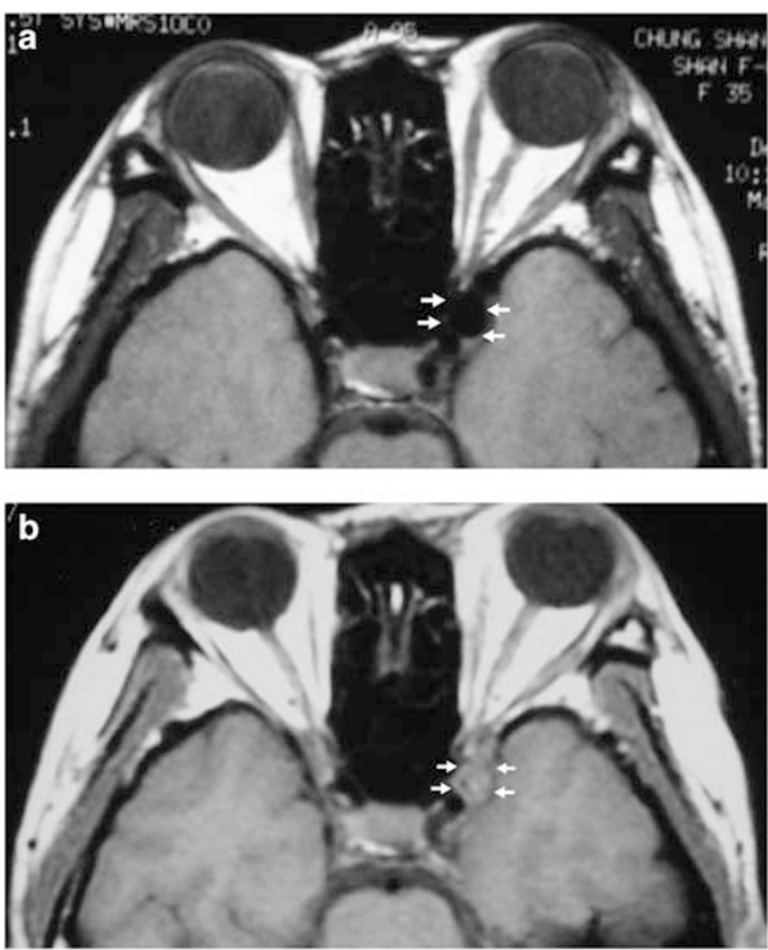

Figure 3 (a) MR T1-weighted image showed abnormal flow void signal in the enlarged left cavernous sinus (arrows) before gamma knife radiosurgery. (b) 1 month after gamma knife radiosurgery, the flow void signal at the anterior portion of the left cavernous sinus was replaced by tissue density (arrows), showing thrombous formation at this portion.

engorged left SOV. The cerebral angiography showed subtotal obliteration of the fistula.

Proptosis of the left eye improved within 1 month and vision recovered to $6 / 6$. MR angiography examination showed complete obliteration of the fistula. Doppler ultrasonography performed 2 years later showed no flow in the left SOV.

\section{Comment}

Acute SOV thrombosis with CRVO is a rare event after gamma knife radiosurgery. Chiou et al. ${ }^{4}$ observed normalization of the reverse pulsatile flow in SOV at months after gamma knife radiosurgery of DAVFCS, indicating a gradual change of haemodynamics. Previous report showed a closure time of 6-15 months for the complete obliteration of the fistula after radiosurgry. ${ }^{2}$ It seems that this early thrombotic episode in our patient is unusual in the treatment course of gamma knife radiosurgery of DAVFCS.

SOV thrombosis had been observed by MRI in patients with paradoxical worsening of symptoms and signs before the onset of clinical improvement. ${ }^{5}$ It has been postulated that the clinical improvement may be due to the extension of the thrombus from SOV to occlude the dural-cavernous shunts, followed by the development of collateral vascular channels. There has been no concrete evidence to support this mechanism. As for our patient, the concurrent thrombus formation in the SOV and in the anterior cavernous sinus provides evidence for the aforementioned hypothesis of thrombus extension, and depicts the mechanism of paradoxical worsening of symptoms and signs before clinical improvement.

In conclusion, SOV thrombosis with paradoxical worsening of symptoms and signs may also occur in DAVFCS after receiving gamma knife radiosurgery. The thrombus formation of the anterior cavernous sinus in our case lends support to the mechanism of the phenomenon that clinical improvement may ensue after SOV thrombosis in DAVFCS.

\section{References}

1 Barcia JL, Soler F, Barcia JA, Hernandez G. Radiosurgery of carotid-cavernous fistula. Acta Neurochir 1994; 62: 10-12.

2 Guo WY, Pan DH, Wu HM, Chung WY, Shiau CY, Wang LW et al. Radiosurgery as a treatment alternative for dural arteriovenous fistulas of the cavernous sinus. AJNR Am J Neuroradiol 1998; 19: 1081-1087.

3 Barrow DL, Spector RH, Braun IF, Landman JA, Tindall SC, Tindall GT. Classification and treatment of spontaneous carotid-cavernous sinus fistulas. J Neurosurg 1985; 62: 248-256.

4 Chiou HJ, Chou YH, Guo WY, Teng MM, Hsu CC, Tiu CM et al. Verifying complete obliteration of carotid arterycavernous sinus fistula: role of color Doppler ultrasonography. J Ultrasound Med 1998; 17: 289-295.

5 Sergott RC, Grossman RI, Savino PJ, Bosley TM, Schatz NJ. The syndrome of paradoxical worsening of dural-cavernous sinus arteriovenous malformations. Ophthalmology 1987; 94: 205-212. 
L-I Lau' ${ }^{1,2}, \mathrm{H}-\mathrm{M} \mathrm{Wu} \mathrm{Wu}^{3,4}, \mathrm{~A}-\mathrm{G}$ Wang ${ }^{1,4}, \mathrm{M}-\mathrm{Y} \mathrm{Yen}^{1,4}$ and W-M Hsu ${ }^{1,4}$

${ }^{1}$ Department of Ophthalmology, Taipei Veterans General Hospital, Taipei, Taiwan

${ }^{2}$ Institute of Clinical Medicine, School of Medicine, National Yang-Ming University, Taipei, Taiwan

${ }^{3}$ Department of Radiology, Taipei Veterans General Hospital, Taipei, Taiwan

${ }^{4}$ Department of Ophthalmology, School of Medicine, National Yang-Ming University, Taipei, Taiwan

Correspondence: A-G Wang, Department of Ophthalmology,

Taipei Veterans General Hospital, National Yang-Ming University,

No. 201, Section 2,

Shih-Pai Road, Shih-Pai,

Taipei 11217, Taiwan

Tel: + 886228757325 ;

Fax: + 886228761351

E-mail: agwang@vghtpe.gov.tw

The authors have no proprietary interest in any aspect of this report

Eye (2006) 20, 1426-1428. doi:10.1038/sj.eye.6702287; published online 17 February 2006

Sir,

Pseudophakic bullous keratopathy in a case of corneal birth trauma

Corneal oedema and bullous keratopathy are known complications of cataract surgery and can occur following an uncomplicated procedure. ${ }^{1}$ Various predisposing factors for pseudophakic bullous keratopathy include pre-existing corneal endothelial disease such as Fuchs dystrophy ${ }^{1}$ and use of anterior chamber and iris-supported lenses. ${ }^{2}$ Birth trauma to the cornea is known to cause a decrease in the density of endothelial cells. ${ }^{3}$ This may cause corneal oedema at birth or at any later stage. ${ }^{3}$ However, we are not aware of any reported cases of corneal oedema following cataract surgery in such eyes. We recently had a patient with a peripheral Descemets membrane rupture due to birth trauma who developed pseudophakic bullous keratopathy following uncomplicated phacoemulsification.

\section{Case report}

A 76-year-old male patient presented with diminution of vision in the right eye due to progressive cataract. His best-corrected visual acuity was 6/12 in the right eye and $6 / 9$ in the left eye. Preoperative assessment revealed a right corneal curvilinear opacity, $2 \mathrm{~mm}$ from the periphery, at the level of the Descemets membrane and endothelium (Figure 1). There was no other abnormality in either eye. Retrospectively, birth history revealed a forceps-assisted delivery. There was no depression on the contra lateral occipital region, a sign that may be present in patients having corneal birth trauma. ${ }^{3}$ Uncomplicated phacoemulsification through a superiorly placed clear corneal incision with in-the-bag implantation of an acrylic lens was performed. The total ultrasound time was $71.03 \mathrm{~s}$ and the average ultrasound power used was $8 \%$. Significant corneal oedema was noted on the first post-operative day and the best-corrected visual acuity was $6 / 36$. A week later the cornea developed some bullae. On the last postoperative visit, 5 months after the surgery, the best-corrected visual acuity was $6 / 12$, with some improvement in the corneal condition. Specular microscopy of the fellow eye revealed normal endothelial cell count and morphology. The corneal oedema made it impossible to perform specular microscopy of the affected eye.

\section{Comment}

Our patient had an unusual corneal pathology, clinically suggestive of the Descemets membrane rupture. In the absence of any other corneal findings, the history of

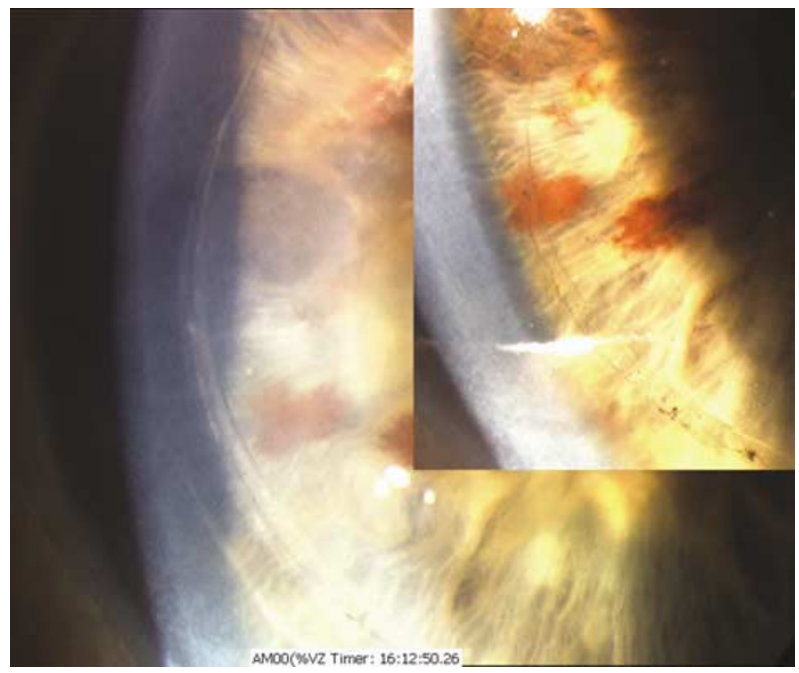

Figure 1 A curvilinear Descemet's membrane split-evidence of corneal birth trauma. (Inset: magnified view under focal illumination.) 